\title{
Treatment of vasospasm in bacterial meningitis with hemodynamic augmentation
}

\author{
Sigmon $\mathrm{JL}^{* *}$, Ball $\mathrm{AM}^{2}$, Namen $\mathrm{AM}^{3}$ and Tucker $\mathrm{KL}^{4}$ \\ ${ }^{1}$ Department of Pharmacy, Duke University Hospital, USA \\ ${ }^{2}$ Clinical Manager, Clinical and Patient Care Services, Department of Pharmacy, Duke University Hospital, USA \\ ${ }^{3}$ Associate Professor of Medicine Pulmonary, Critical Care, Allergy \& Immunology, Wake Forest Baptist Health, USA \\ ${ }^{4}$ Assistant Professor, Department of Neurology and Anesthesiology-Critical Care, Wake Forest Baptist Health, USA
}

\begin{abstract}
Introduction Cerebrovascular complications occur in $15-20 \%$ of patients with acute bacterial meningitis infections and are associated with a poor prognosis. Arterial narrowing is the most common finding, most likely resulting from inflammation associated with meningitis. We report neurologic recovery from hemodynamic management in a patient with meningitis associated vasospasm.

Observation A 43 year old female was admitted to a medical intensive care unit with fever, right ear pain with drainage, and seizures. Brain imaging revealed bilateral otomastoiditis, right temporal cerebritis, and right transverse sinus thrombosis requiring anticoagulation. Vancomycin, metronidazole, and ceftriaxone were initiated for meningitis followed by mastoidectomy. Prior to surgery, a head and neck computer tomography angiography revealed diffuse decreased caliber of the intracranial vessels, concerning for vasospasm. Transcranial Doppler ultrasound confirmed cerebral artery vasospasm. Neurology consultants recommended conservative hemodynamic augmentation given risk for bleeding with venous sinus thrombosis with a goal systolic blood pressure (SBP) of 120 - $140 \mathrm{mmHg}$ and mean arterial pressure (MAP) no greater than 100. Phenylephrine was initiated, although nicardipine was later required to maintain target SBP goal. After initiation of SBP augmentation within 12 hours, the patient's Glasgow Coma Scale (GCS) score improved from 5 to 10 . Within several weeks, the patient was discharged with significant neurological recovery only limited by global aphasia which recovered at follow-up.

Conclusion and relevance Hemodynamic augmentation therapy is an important treatment strategy for vasospasm in patients with subarachnoid hemorrhage (SAH) with the goal of improving cerebral perfusion to overcome the increased vascular resistance in the cerebral arteries. This case demonstrates meningitis associated vasospasm can also be mitigated by augmentation of SBP in an effort to increase cerebral perfusion and minimize neurologic injury. Neurologic improvement occurred after initiation of hemodynamic augmentation in this patient with severe vasospasm induced by inflammation from meningitis.
\end{abstract}

\section{Manuscript}

Cerebrovascular complications occur in $15-20 \%$ of patients with acute bacterial meningitis infections and are associated with a poor prognosis [1]. Arterial narrowing is the most common finding, resulting most likely from inflammation associated with meningitis [2]. Currently, there is a paucity of data on the treatment of severe vasospasm associated with meningitis. We report neurologic recovery from hemodynamic management in a patient with meningitis associated vasospasm.

\section{Report of case}

A 43-year-old female presented one month after a posterior lumbar fusion at L5-S1 and L4-L5 with altered mental status requiring intubation, fever, and several days of right ear purulent drainage. She had a history for seizure-like episodes for which she did not take medication and a remote history of cocaine use.

On physical exam, she was intubated with non-purposeful movements, although withdrawing from painful stimuli.

Head computed tomography imaging revealed bilateral mastoid and middle ear effusions and a small pneumocephalus in right middle cranial fossa concerning for possible epidural abscess or subdural empyema. Otolaryngology placed a right myringotomy with tympanostomy tube. Neurosurgery then performed a lumbar puncture (results listed in Table 1). Brain magnetic resonance imaging was significant for bilateral meningitis complicated by intracranial extension and an area of cerebritis along the right temporal lobe measuring $2.0 \times 2.0$ centimeters. A focal filling defect was also noted within the right transverse sinus, consistent with dural venous sinus thrombosis that was treated initially with heparin infusion and later transitioned to warfarin via enoxaparin bridge.

Despite patient's confusion, the initial neurologic examinations were nonfocal. On day two of admission, the patient demonstrated new right upper extremity weakness and upgoing toe. Throughout the remainder of her course, neurologic examinations were remarkable for left gaze preference, right sided upper extremity weakness, right upgoing toe, and global aphasia.

Correspondence to: Sigmon JL, Department of Pharmacy, Duke University Hospital, 1422 Duke South, Trent Drive, DUMC Box 3089, Durham, NC 27710 , USA, Tel: 919-681-6344; E-mail: jana.sigmon@duke.edu

Key words: cerebral ischemia, meningitis, vasospasm

Received: November 03, 2017; Accepted: November 21, 2017; Published: November 25, 2017 
Vancomycin, metronidazole, and ceftriaxone were initiated as empiric antibiotics at admission and narrowed to long term intravenous ceftriaxone based on infectious disease consultant recommendations and culture data results. Cerebrospinal fluid, middle ear fluid, and blood cultures were all positive for Streptococcus pneumoniae. On day five of admission, a mastoidectomy was performed due to lack of improvement with appropriate antimicrobial therapy and persistent mastoiditis on imagining. Head and neck computed tomography angiography before surgery revealed diffuse decreased caliber of the intracranial vessels, concerning for vasospasm. Transcranial Doppler ultrasound (TCD) revealed increased velocity suggesting severe vasospasm in the left middle cerebral artery (MCA), moderate in the right MCA, and left anterior cerebral artery (ACA), and mild in the right ACA. TCD mean velocity results listed in Table 2.

Due to evidence of vasospasm on TCD and cerebral hypoperfusion on electroencephalography, neurology consultants recommended conservative hemodynamic augmentation given risk for bleeding with venous sinus thrombosis with a goal systolic blood pressure (SBP) of $120-140 \mathrm{mmHg}$ and mean arterial pressure (MAP) no greater than 100. Phenylephrine infusion was titrated to goal SBP for 15 hours. Neurologic improvement occurred within 12 hours of initiating phenylephrine as evidenced by the Glasgow Coma Scale (GCS) score increasing from 5 to 10 . After 15 hours, phenylephrine infusion was turned off and a nicardipine infusion was subsequently started to maintain a normotensive SBP less than $160 \mathrm{mmHg}$ and MAP less than 100 to avoid risk of intracerebral hemorrhage. The patient continued to improve neurologically, maintaining a GCS score of 10 thereafter.

Nine days later a repeat TCD showed marked improvement. Bilateral MCA and ACA velocities were reduced by half. At discharge, she was able to follow commands, verbalize, and track to visual stimuli. Overall, she made significant neurologic improvement throughout her stay, only limited by global aphasia [Figure 1 and 2].

\section{Discussion}

Cerebrovascular complications due to arterial narrowing as evidenced by increased cerebral blood flow velocity (CBFv) greater than $150 \mathrm{~cm} / \mathrm{s}$ on TCD is associated with an increased risk of stroke and poor functional outcomes [2]. We are unaware of any randomized, controlled trials evaluating treatment strategies for vasospasm in acute bacterial meningitis secondary to otomastoiditis. Case reports and retrospective analyses have supported aggressive interventions including intra-arterial vasodilator therapy, suboccipital decompression, intracranial bypass, barbiturate coma, and therapeutic

Table 1. Cerebrospinal Fluid Data*

\begin{tabular}{|c|c|}
\hline WBC & $\begin{array}{c}3250(0-10 / \mathrm{mm} 3) \\
97 \% \text { polymorphonuclear cells }\end{array}$ \\
\hline RBC & $233(0 / \mathrm{mm} 3)$ \\
\hline Glucose & $<10 \mathrm{mg} / \mathrm{dL}$ \\
\hline Protein & $552(15-45 \mathrm{mg} / \mathrm{dL})$ \\
\hline Lactate & $13.9(1.1-2.8 \mathrm{mmol} / \mathrm{L})$ \\
\hline
\end{tabular}

Table 2. Transcranial Doppler Mean Velocities*

\begin{tabular}{|c|c|}
\hline Left MCA $\dagger$ & $213(40-80 \mathrm{~cm} / \mathrm{sec})$ \\
\hline Right MCA & $146(40-80 \mathrm{~cm} / \mathrm{sec})$ \\
\hline Left ACA $\dagger$ & $125(35-60 \mathrm{~cm} / \mathrm{sec})$ \\
\hline Right ACA & $106(25-60 \mathrm{~cm} / \mathrm{sec})$ \\
\hline
\end{tabular}

*Transcranial doppler mean velocities (reference ranges),

$\dagger \mathrm{MCA}=$ middle cerebral artery,

$\$ \mathrm{ACA}=$ anterior cerebral artery
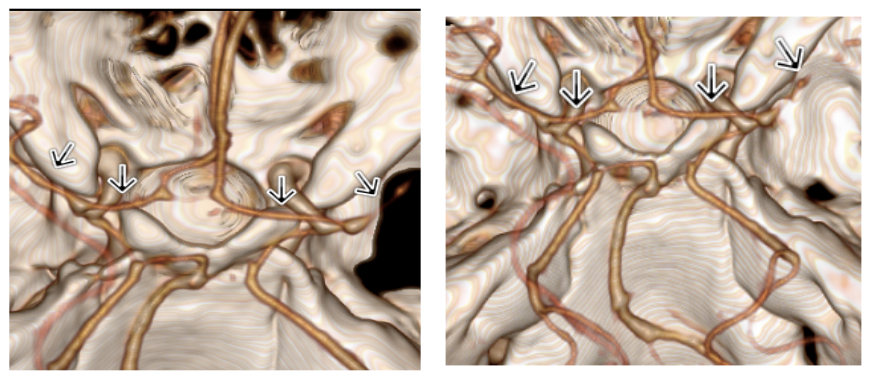

Figure 1. Circle of Willis 3D volume rendering processed from computed tomography angiography of the head and neck. Vasospasm is evidenced by diminished vessel of the vessels, notably the A1 and M1 segments of the anterior and middle cerebral arteries (arrows).
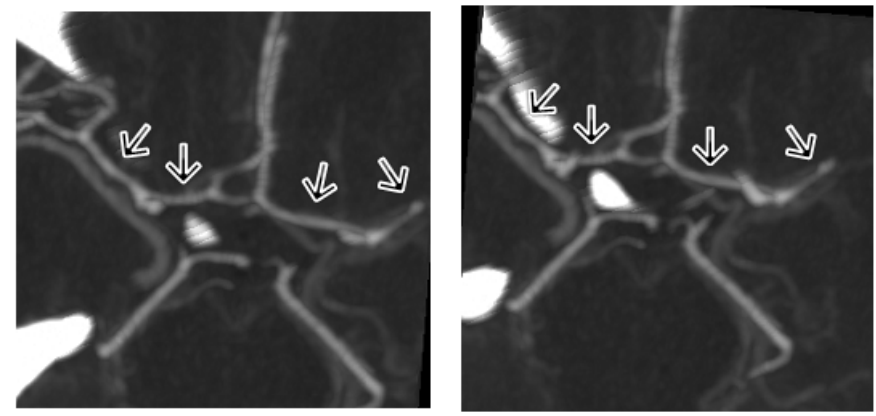

Figure 2. Circle of Willis maximum intensity projection processed from computed tomography angiography of the head and neck. Vasospasm is evidenced by diminished caliber of the vessels, notably the A1 and M1 segments of the anterior and middle cerebral arteries (arrows)

hypothermia $[3,4]$. However, few if any, prospective trials are available that can guide our treatment strategies for vasospasm in acute bacterial meningitis.

Hemodynamic augmentation therapy is an important treatment strategy for vasospasm in patients with subarachnoid hemorrhage (SAH) with the goal of improving cerebral perfusion to overcome the increased vascular resistance in the cerebral arteries [5]. This case demonstrates meningitis associated vasospasm can also be mitigated by augmentation of SBP in an effort to increase cerebral perfusion and minimize neurologic injury.

\section{Conclusion}

Hemodynamic augmentation may have a role in the treatment of cerebrovascular complications of bacterial meningitis such as vasospasm by increasing cerebral perfusion pressure. Neurologic improvement occurred after initiation of hemodynamic augmentation in this patient with severe vasospasm induced by inflammation from meningitis. Further studies should evaluate hemodynamic augmentation therapy in patients with cerebrovascular complications in bacterial meningitis.

\section{Acknowledgements}

Byron A. Feig, MD, MBA, PGY-5 Department of Radiology, Wake Forest Baptist Medical Center

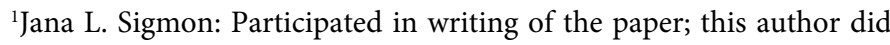
not receive any financial or other forms of support related to this study; this author has no actual or potential conflict of interest in relation to this study 
${ }^{2}$ Amanda M. Ball: Participated in writing of the paper; this author did not receive any financial or other forms of support related to this study; this author has no actual or potential conflict of interest in relation to this study

${ }^{3}$ Andrew M. Namen: Participated in writing of the paper; this author did not receive any financial or other forms of support related to this study; this author has no actual or potential conflict of interest in relation to this study

${ }^{4}$ Kristi L. Tucker: Participated in of the paper; this author did not receive any financial or other forms of support related to this study; this author has no actual or potential conflict of interest in relation to this study

\section{References}

1. Chow FC, Marra CM, Cho TA (2011) Cerebrovascular disease in central nervous system infections. Semin Neurol 31: 286-306. [Crossref]

2. Klein M, Koedel U, Pfefferkorn T, Zeller G, Woehrl B, et al. (2011) Arterial cerebrovascular complications in 94 adults with acute bacterial meningitis. Crit Care 15: R281. [Crossref]

3. Nussbaum ES, Lowary J, Nussbaum LA (2015) A multidisciplinary approach to the treatment of severe cerebral vasospasm following bacterial meningitis: a case report and literature review. Surg Neurol Int 6: 148. [Crossref]

4. Taqui A, Koffman L, Hui F, Gomes J, Hussain MS, et al. (2014) Intra-arterial vasodilator therapy for parainfectious cerebral vasospasm. J Neurol Sci 340: 225-229. [Crossref]

5. Rabinstein AA, Lanzino G, Wijdicks EF (2010) Multidisciplinary management and emerging therapeutic strategies in aneurysmal subarachnoid hemorrhage. Lancet Neurol 9: 504-519. [Crossref]

Copyright: (C2017 Sigmon JL. This is an open-access article distributed under the terms of the Creative Commons Attribution License, which permits unrestricted use, distribution, and reproduction in any medium, provided the original author and source are credited. 